\title{
Postsynaptic Targets of Purkinje Cell Terminals in the Cerebellar and Vestibular Nuclei of the Rat
}

\author{
C. I. De Zeeuw ${ }^{1}$ and A. S. Berrebi ${ }^{2}$ \\ 'Department of Anatomy, Erasmus University Rotterdam, PO Box 1738, 3000 DR, Rotterdam, The Netherlands \\ 2Departments of Otolaryngology and Anatomy, PO Box 9200 HSCS, Morgantown, WV 26506-9200, USA
}

Keywords: nucleus prepositus hypoglossi, gap junctions, L7, GABA, glycine, primary afferents, cerebellum

\begin{abstract}
The cerebellar and vestibular nuclei consist of a heterogeneous group of inhibitory and excitatory neurons. A major proportion of the inhibitory neurons provides a GABAergic feedback to the inferior olive, while the excitatory neurons exert more direct effects on motor control via non-olivary structures. At present it is not clear whether Purkinje cells innervate all types of neurons in the cerebellar and vestibular nuclei or whether an individual Purkinje cell axon can innervate different types of neurons. In the present study, we studied the postsynaptic targets of Purkinje cell axons in the rat using a combination of pre-embedding immunolabelling of the Purkinje cell terminals by L7, a Purkinje cell-specific marker, and postembedding GABA and glycine immunocytochemistry. In the cerebellar nuclei, vestibular nuclei and nucleus prepositus hypoglossi Purkinje cell terminals were found apposed to GABAergic and glycinergic neurons as well as to larger non-GABAergic, nonglycinergic neurons. In the cerebellar and vestibular nuclei individual Purkinje cell terminals innervated both the inhibitory and excitatory neurons. Both types of neurons were contacted not only by GABAergic Purkinje cell terminals but also by GABA-containing terminals that were not labelled for $L 7$ and by non-GABAergic, nonglycinergic terminals that formed excitatory synapses. Glycine-containing terminals were relatively scarce $(<2 \%$ of the GABA-containing terminals) and frequently contacted the larger non-GABAergic, non-glycinergic neurons. To summarize, Purkinje cell axons evoke their effects through different types of neurons present in the cerebellar and vestibular nuclear complex. The observation that individual Purkinje cells can innervate both excitatory and inhibitory neurons suggests that the excitatory cerebellar output system and the inhibitory feedback to the inferior olive are controlled simultaneously.
\end{abstract}

\section{Introduction}

The cerebellar and vestibular nuclei form the output of the cerebellum. This complex of nuclei consists of a heterogeneous group of excitatory and inhibitory projection neurons and interneurons. Most of the excitatory neurons are larger and use glutamate and/or aspartate as their neurotransmitter, while the inhibitory neurons are mostly medium-sized or small and use GABA and/or glycine as their neurotransmitter (Nelson and Mugnaini, 1989; Walberg et al., 1990; Kevetter and Hoffman, 1991; Batini et al., 1992; Chen and Hillman, 1993). The excitatory projection neurons in the cerebellar nuclei innervate a wide variety of areas, including the thalamus, mesodiencephalic junction, pontine and reticular nuclei, medulla oblongata and spinal cord (Fukushima et al., 1977; Wilson et al., 1977; De Zeeuw and Ruigrok, 1994; for review see Ito, 1984), while the GABAergic neurons innervate predominantly the inferior olive (De Zeeuw et al., 1989; Nelson and Mugnaini, 1989; Fredette and Mugnaini, 1991). In addition, some GABAergic cerebellar nuclei neurons may give rise to projections to the cerebellar cortex (Batini et al., 1992) or pons (Aas and Brodal, 1990). Even though most of the glycinergic neurons in the cerebellar nuclei have been reported to be GABAergic (Chen and Hillman, 1993), none of them has been found to project to the inferior olive (De Zeeuw et al., 1994a). The excitatory and inhibitory projection neurons in the vestibular complex innervate the oculomotor complex (Spencer et al., 1989, 1992; Wentzel et al., 1995), inferior olive (Barmack et al., 1989; De Zeeuw et al., 1993) and spinal cord (Shinoda et al., 1992). With regard to the inhibitory vestibular neurons, GABA is employed for the innervation of the oculomotor nucleus and inferior olive, while glycine is probably the inhibitory neurotransmitter for the innervation of the abducens nucleus and spinal cord.

Despite the extensive knowledge on these different types of neurons in the cerebellar and vestibular nuclei, it is not known whether Purkinje cells innervate all types of neurons in these nuclei or whether an individual Purkinje cell axon can innervate different types of neurons. Recently, we made biocytin injections into the rabbit vestibulocerebellum to study the branching and termination patterns of individual Purkinje cell axons (De Zeeuw et al., 1994b). Purkinje cell axons were frequently found to branch and innervate more than one vestibular nucleus or to innervate both a cerebellar and a

Correspondence to: Dr C. I. De Zeeuw, as above 
vestibular nucleus. Light microscopic analysis of the labelled terminal varicosities of the branches showed that an individual Purkinje cell axon can be apposed to cells with a wide range of sizes and shapes. Since the smaller and larger cells are usually inhibitory and excitatory respectively (Walberg et al., 1990; Fredette and Mugnaini, 1991; Batini et al., 1992; Chen and Hillman, 1993), it seems likely that an individual Purkinje cell axon can target both inhibitory and excitatory neurons. This pattern of innervation is supported by the observation that many Purkinje cell axons from the flocculus give off terminals both in the dorsolateral and central parts of the superior vestibular nucleus (De Zeeuw et al., 1994b), which are believed to contain excitatory and inhibitory second-order vestibular neurons respectively (Highstein, 1973; Ito et al., 1976; Yamamoto et al., 1978; Highstein and Resine, 1979).

To determine whether individual Purkinje cells establish synaptic contacts with both excitatory and inhibitory neurons, we studied the postsynaptic targets of Purkinje cell axons in the cerebellar and vestibular nuclei of the rat at the electron microscope level using a combination of postembedding GABA or glycine immunocytochemistry with pre-embedding L7 immunolabelling of Purkinje cell terminals. The antibody against the $\mathrm{L} 7$ protein is an ideal marker because L7 occurs in the brain exclusively in Purkinje cells, is expressed by all Purkinje cells, and is distributed in all neuronal components, including axon terminals (Oberdick et al., 1988; Berrebi and Mugnaini, 1992).

\section{Materials and methods}

\section{Antisera}

Production of the rabbit $\mathrm{L} 7$ antiserum used in this study has been described in detail by Oberdick et al. (1988) and Berrebi et al. (1991); it was kindly provided by Dr J. I. Morgan. Briefly, L7 antiserum was raised against a peptide corresponding to amino acids 41-53 of the predicted L7 sequence (MDDQRVTVNSLPG) coupled to bovine thyroglobulin with glutaraldehyde. The GABA and glycine antisera were generously supplied by Drs R. M. Buijs and R. J. Wenthold respectively; the sensitivity and specificity of both antisera have been tested extensively (Seguela et al., 1984; Buijs et al., 1987; Wenthold et al., 1987; De Zeeuw et al., 1989). Immunopress-blot analysis, in which the same antibody concentrations were used as in the immunocytochemistry, indicated that cross-reactivities of the GABA antibody with glycine and of the glycine antibody with GABA were very low. The GABA antibody detected GABA and glycine at concentrations of 1.1 and $1000 \mathrm{ng} / \mu \mathrm{l}$ respectively, while the glycine antibody detected GABA and glycine at concentrations of 1000 and $3.3 \mathrm{ng} / \mu \mathrm{l}$ respectively.

\section{Light microscopy}

To reveal the general distribution of L7 in the cerebellar nuclei and vestibular complex, rats were processed for light microscopy. The rats were anaesthetized with Nembutal $(60 \mathrm{mg} / \mathrm{kg}$, i.v.), and perfused transcardially with $250 \mathrm{ml} 0.9 \% \mathrm{NaCl}$. Fixation was accomplished with a zinc-aldehyde fixative composed of $4 \%$ formaldehyde (Fisher Scientific), $0.5 \%$ zinc dichromate (K \& K Rare and Fine Chemicals, ICN), $0.125 \%$ glutaraldehyde (TAAB, Marivac) and $0.75 \% \mathrm{NaCl}$, $\mathrm{pH} 4.8\left(20 \mathrm{ml}, 20^{\circ} \mathrm{C}\right)$. One hour after perfusion the brains were dissected from the cranium and cryoprotected in $30 \%$ sucrose in $0.9 \% \mathrm{NaCl}$ for 2 days at $6^{\circ} \mathrm{C}$.

Frozen tissue blocks were cut in $30 \mu \mathrm{m}$ transverse sections on a

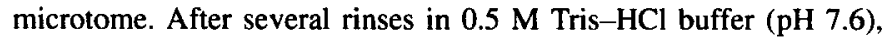

free floating sections were immunoreacted in tissue culture wells using the peroxidase-antiperoxidase procedure according to the following protocol: $\mathrm{I} \mathrm{h}$ incubation in 5\% normal donkey serum (NDS) with $0.5 \%$ Triton $\mathrm{X}-100 ; 40 \mathrm{~h}$ incubation at $4^{\circ} \mathrm{C}$ in the polyclonal anti-L7 antiserum, diluted $1: 1000$ with $1 \%$ normal donkey serum and $0.2 \%$ Triton X-100; $1 \mathrm{~h}$ incubation in goat anti-rabbit antiserum diluted $1: 100$, followed by rabbit peroxidase-antiperoxidase antiserum, diluted 1:100, each with $1 \%$ normal donkey serum and $0.2 \%$ Triton $\mathrm{X}-100$. The antibody-antigen binding sites were revealed by incubation in $0.05 \%$ diaminobenzidine and $0.01 \% \mathrm{H}_{2} \mathrm{O}_{2}$. All immunoreagent solutions were diluted in $0.5 \mathrm{M}$ Tris- $\mathrm{HCl}$ buffer $(\mathrm{pH} 7.6)$, which also served as the rinsing solution between each step of the immunoreaction. Control sections incubated with preimmune serum instead of primary antiserum were free of immunoreaction product.

The light microscopic distribution of GABAergic and glycinergic neurons and terminals will not be demonstrated in the present paper (for details see Nelson and Mugnaini, 1989; Walberg et al., 1990; Chen and Hillman, 1993).

\section{Electron microscopy}

Four adult rats were processed for pre-embedding L7 immunocytochemistry and postembedding GABA and glycine immunocytochemistry. The rats were anaesthetized with Nembutal $(60 \mathrm{mg} / \mathrm{kg}$, i.v.) and perfused transcardially with $100 \mathrm{ml}$ of a calcium-free variant of Ringer solution, saturated with $95 \% \mathrm{O}_{2}$ and $5 \% \mathrm{CO}_{2}$ to $\mathrm{pH} 7.2$, and 1 litre of fixative consisting of $2 \%$ glutaraldehyde and $2 \%$ paraformaldehyde in $0.12 \mathrm{M}$ sodium phosphate buffer ( $\mathrm{pH} \mathrm{7.3)} \mathrm{at}$ $20^{\circ} \mathrm{C}$. One hour after perfusion the brains were removed from the calvaria, sectioned coronally on a Vibratome at $40 \mu \mathrm{m}$, and collected

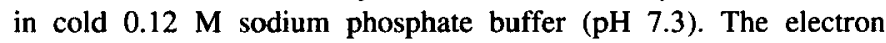
microscopic immunocytochemistry protocol for $\mathrm{L} 7$ was as for the light microscopy described above, except that (i) the Triton X-100 detergent was omitted; (ii) Tris-buffered saline ( 295 mOsm) was used as the diluent and rinsing solution; and (iii) incubation in the primary antiserum took place overnight at room temperature.

After the L7 immunoreaction, the sections were rinsed in $0.12 \mathrm{M}$ sodium phosphate buffer $(\mathrm{pH} 7.3$ ), postfixed for $1 \mathrm{~h}$ in $2 \%$ buffered osmium tetroxide in $8 \%$ D-glucose, rinsed thoroughly in distilled water, stained in $2 \%$ aqueous uranyl acetate, rinsed in distilled water, dehydrated in a graded series of ethanols and propylene oxide, and flat-embedded in Araldite.

Subsequently, we prepared pyramids of the cerebellar and vestibular nuclei and nucleus prepositus hypoglossi and cut ultrathin sections from the most superficial part (a layer of $\sim 3 \mu \mathrm{m}$ ) of these pyramids, which contained abundant L7 labelling. The ultrathin sections were mounted on Formvar-coated nickel grids, and processed for GABA or glycine immunocytochemistry (for details see De Zeeuw et al., 1989, 1994a). Briefly, grids were rinsed in a solution of Tris buffer containing $0.9 \% \mathrm{NaCl}$ and $0.1 \%$ Triton $\mathrm{X}-100$ at $\mathrm{pH} 7.6$ (TBST), and alternately incubated overnight in a droplet of GABA (1:1000 in TBST) or glycine (1:250 in TBST) antiserum. The next day the grids were rinsed twice with TBST and stored for $30 \mathrm{~min}$ in the rinsing solution. After rinsing with TBST (pH 8.2) the grids were incubated for $1 \mathrm{~h}$ in a droplet of goat anti-rabbit IgG labelled with $15 \mathrm{~nm}$ gold particles (Janssen Pharm.) diluted 1:25 in TBST. Subsequently, the grids were washed twice with TBST $(\mathrm{pH} \mathrm{7.6)}$ and distilled water, counterstained with uranyl acetate and lead citrate, and examined in a Philips electron microscope. A neuronal structure was considered as GABA- or glycine-positive when the number of gold particles overlying it was at least eight times higher than the number of particles overlying an equal surface area of surrounding glial structures 


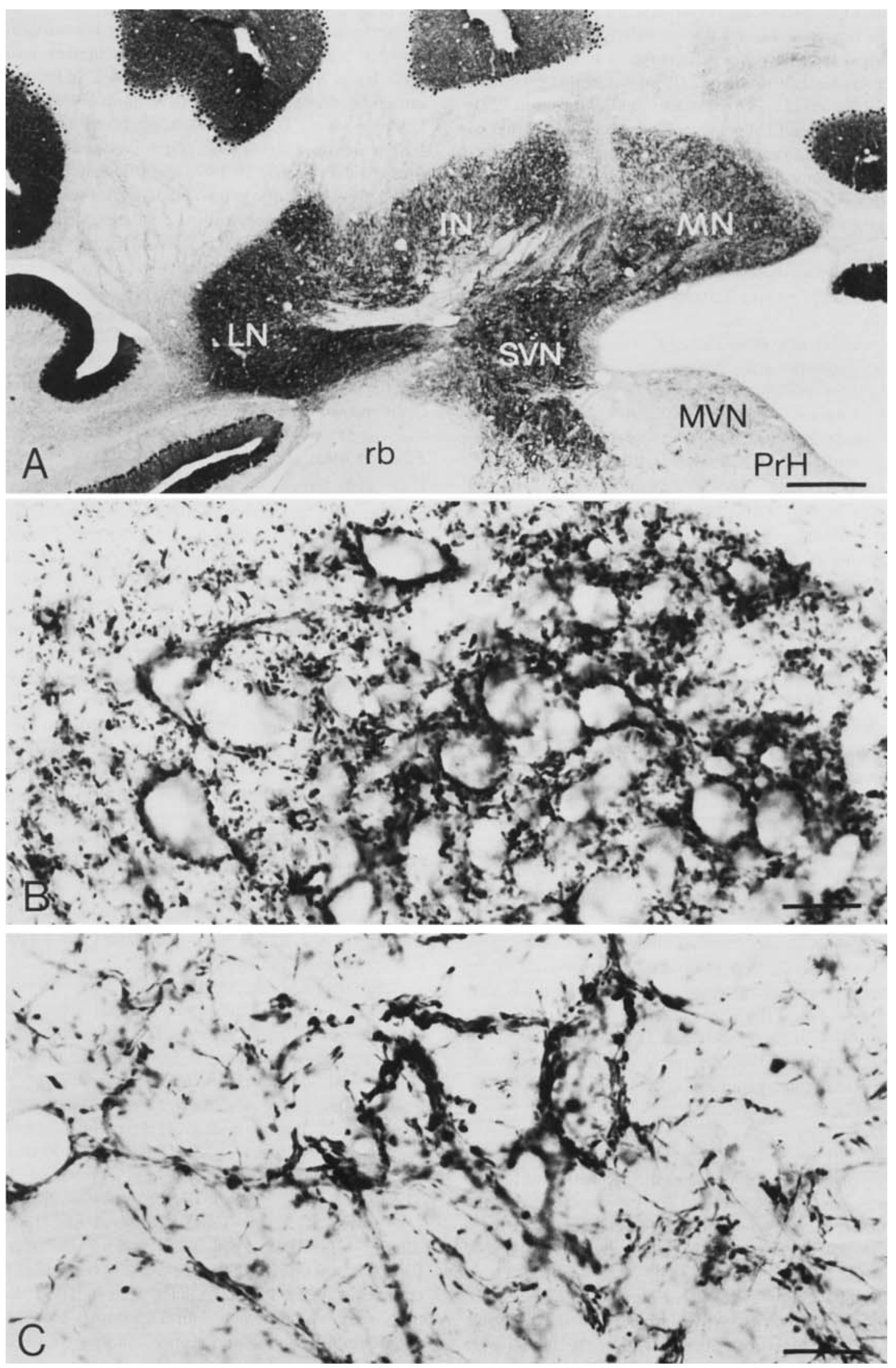

FIG. 1. Micrographs of the cerebellar and vestibular nuclei of sections immunostained for L7. (A) The density of immunoreactive terminals in the lateral cerebellar nucleus (LN), interposed cerebellar nucleus (IN), medial cerebellar nucleus (MN) and superior vestibular nucleus (SVN) is higher than in the medial vestibular nucleus (MVN) and the nucleus prepositus hypoglossi (PrH). (B, C) Examples of L7-labelled boutons in the IN and PrH respectively. Scale bars: A, $390 \mu \mathrm{m} ; \mathrm{B}, 23 \mu \mathrm{m} ; \mathrm{C}, 16 \mu \mathrm{m}$. 

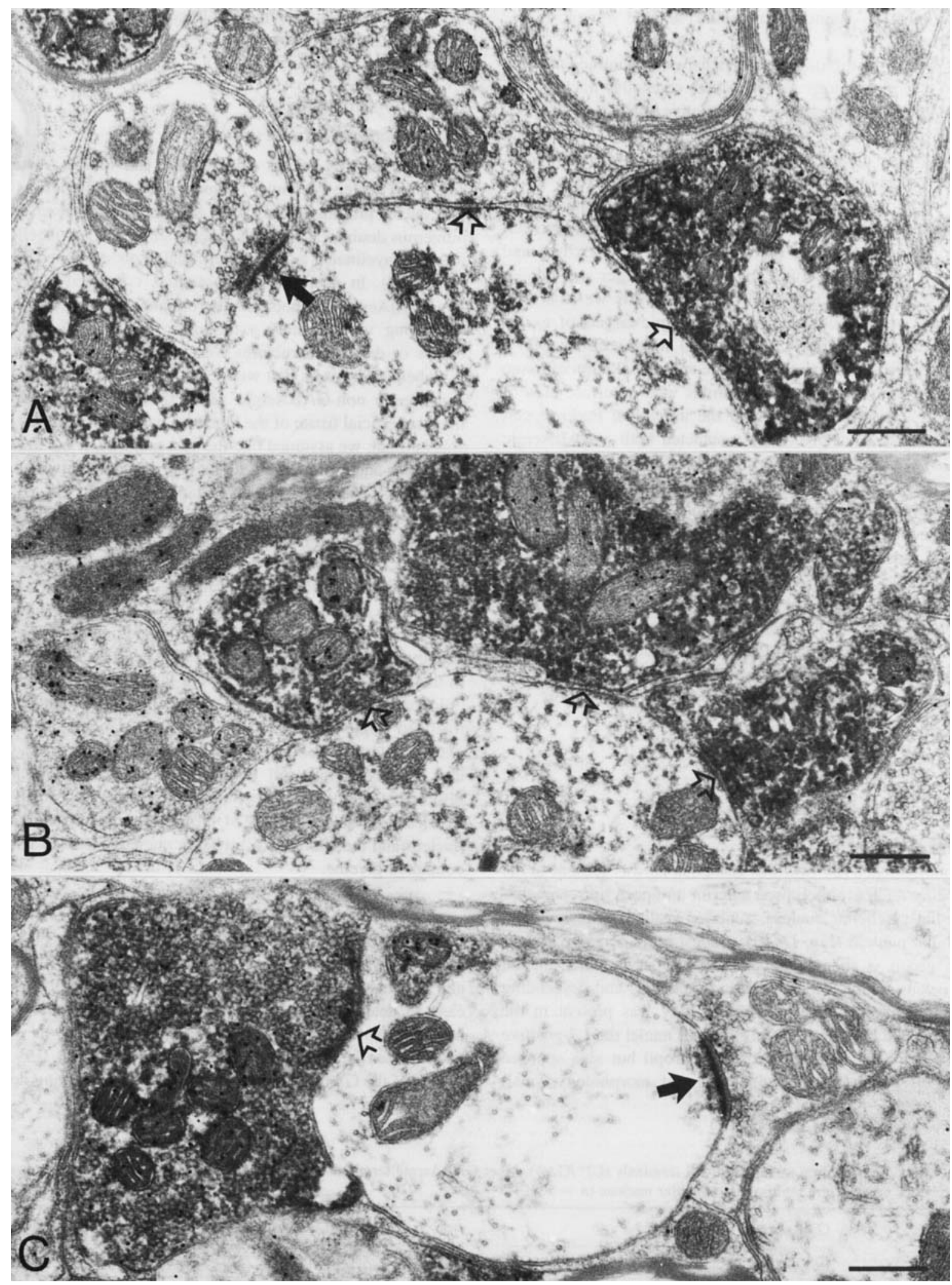

FIG. 2. Electron micrographs of GABAergic, L7-labelled Purkinje cell terminals, GABAergic terminals that are not labelled for L7, and non-labelled terminals in the lateral cerebellar nucleus (A), superior vestibular nucleus (B) and nucleus prepositus hypoglossi (C). Note that the L7-labelled terminals can be readily distinguished from the GABAergic terminals that are not labelled for L7, and that the dendrites in all micrographs are contacted by different types of terminals, indicating that individual neurons in the cerebellar and vestibular nuclei receive both GABAergic terminals from Purkinje cell axons and GABAergic terminals from interneurons or neurons that project to the inferior olive. Note also that the Purkinje cell terminals in these electron micrographs cannot be distinguished from the other GABAergic terminals on morphological criteria. Open arrows indicate symmetrical synapses and filled arrows indicate asymmetric synapses. The asymmetrical synapses frequently showed subsynaptic densities known as Taxi bodies. Scale bars: A, $0.34 \mu \mathrm{m} ; \mathrm{B}, 0.39 \mu \mathrm{m} ; \mathrm{C}, 0.28 \mu \mathrm{m}$. 
(for details about the counting procedure see De Zeeuw et al., 1989, 1994a).

The postsynaptic distribution was extensively quantified in both non-serial and serial sections of the lateral cerebellar nucleus and interposed cerebellar nucleus. In the quantitative analysis of the nonserial sections samples of GABA-containing terminals (i.e. including those labelled for L7) and non-GABAergic terminals were taken from at least three ultrathin sections obtained from at least two tissue blocks from each rat. Distinction was made between terminals that formed synaptic contacts with GABAergic and non-GABAergic cell bodies, proximal dendrites and distal dendrites. The proximal and distal dendrites were characterized by a diameter larger and smaller than $2 \mu \mathrm{m}$ respectively. In the serial section analysis we examined the GABAergic and non-GABAergic postsynaptic targets of exclusively the L7 labelled terminals. Sets of $\sim 50$ serial sections with a silver interference colour were cut from the superficial parts of tissue blocks that were trimmed down to pyramids with a surface area of $0.5-0.5 \mathrm{~mm}$. It was determined for all the individual Purkinje cell axons and terminals what percentage contacted both a GABAergic and a non-GABAergic structure.

In addition, we determined the diameters of cell bodies of the GABAergic, glycinergic and non-labelled neurons in both semithin and uitrathin sections. In this analysis only cell bodies with nucleoli were measured (see also Batini et al., 1992).

Finally, colocalization of GABA and glycine in the cerebellar and vestibular nuclei was studied and observed in serial sections that were processed alternately for GABA and glycine immunocytochemistry. However, since the coexistence of these neurotransmitters in these nuclei has already been firmly demonstrated (Walberg et al., 1990; Chen and Hillman, 1993), no further results on this subject will be reported.

\section{Results}

\section{Light microscopy}

Dense L7 immunoreactivity of fibres and boutons was present in the lateral cerebellar nucleus, interposed anterior and posterior cerebellar nucleus, medial cerebellar nucleus, superior vestibular nucleus and lateral vestibular nucleus (Fig. 1A, B); modest immunoreactivity was observed in group $\mathrm{y}$, the magnocellular and parvicellular medial vestibular nucleus, caudal medial vestibular nucleus and descending vestibular nucleus, and weak immunoreactivity was present in the nucleus prepositus hypoglossi (Fig. 1C). In all nuclei the L7-positive boutons were not only distributed in the neuropil but also apposed to neuronal cell bodies with a wide range of morphology, small
(5-15 $\mu \mathrm{m}$ in diameter) to large $(15-25 \mu \mathrm{m})$, spherical or elliptical, triangular or fusiform.

\section{Electron microscopy}

\section{Diversity of labelled structures}

Ultrathin sections of the superficial $3 \mu \mathrm{m}$ of the tissue blocks of cerebellar nuclei, vestibular nuclei and nucleus prepositus hypoglossi that were processed for GABA immunocytochemistry included numerous double L7/GABA-labelled, single GABA-labelled and nonlabelled myelinated axons, preterminal axon segments, and terminals (Figs 2-5). In addition, these sections contained GABAergic and non-GABAergic cell bodies and proximal dendrites and some GABAcontaining unmyelinated axons and peripheral dendrites. No cell bodies or dendritic structures were positive for $\mathrm{L} 7$, and none of the L7 labelled terminals that were examined through serial sections was consistently non-GABAergic. Since the analysis was limited to the most superficial tissue of the blocks in which the antibody penetration was optimal, we assumed that the vast majority, if not all, of Purkinje cell axons and terminals would be L7-immunoreactive; i.e. if GABAcontaining terminals were L7-negative (e.g. Fig. 2A, B), they were interpreted as terminals that were not derived from Purkinje cells.

Quantitatively, the Purkinje cell boutons dominated the neuropil (Table 1): of the terminals collected from the most superficial sections of the lateral cerebellar nucleus and the interposed cerebellar nucleus, $986(73 \%)$ contained GABA and L7, $215(16 \%)$ contained GABA but were L7-negative, and 149 (11\%) were non-GABAergic and L7negative [Palkovits et al. (1977) calculated that $62 \%$ of the boutons in the central nuclei of the cat were Purkinje cell terminals].

Ultrathin sections processed for glycine immunocytochemistry included single L7-labelled, single glycine-labelled and non-labelled axons, preterminal axon segments, and terminals (Fig. 5). Glycinergic and non-glycinergic cell bodies and proximal dendrites were also observed. Overall glycine-containing structures were relatively scarce. In a random selection of 374 terminals from the lateral cerebellar nucleus and the interposed cerebellar nucleus, seven (1.9\%) terminals contained glycine.

\section{Characteristics of cell bodies}

The identity of the cell bodies and proximal dendrites in the cerebellar and vestibular nuclei and nucleus prepositus hypoglossi was usually easy to determine by the presence or absence of the immunogold labelling. Apart from the medial cerebellar nucleus, where glycinergic neurons can be large (see also Chen and Hillman, 1993), the cell bodies of the GABA-containing and glycine-containing neurons were

TABLE 1. Postsynaptic distribution of Purkinje cell terminals $\left(\mathrm{L7}^{+} / \mathrm{GA}^{+}\right)$, other GABAergic terminals $\left(\mathrm{L7}^{-} / \mathrm{GA}^{+}\right)$, and non-GABAergic terminals $\left(\mathrm{L} 7^{-} / \mathrm{GA}^{-}\right)$in the lateral cerebellar nucleus and interposed cerebellar nucleus $(n=1350)$

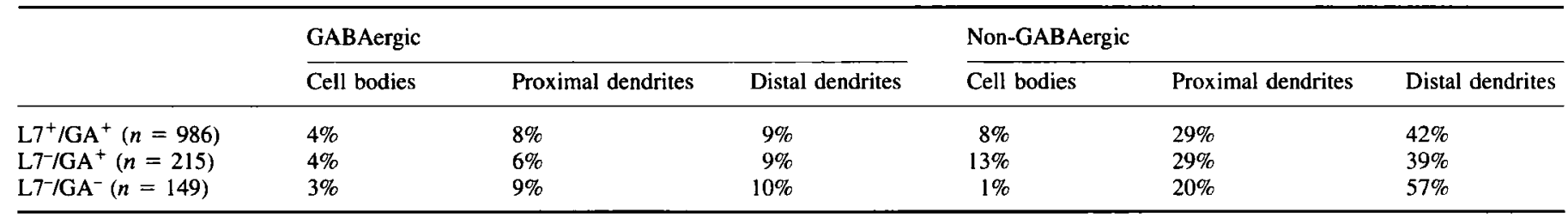

Postsynaptic structures are divided into GABAergic and non-GABAergic cell bodies, proximal dendrites and distal dendrites. The percentages make $100 \%$ for each row. Percentages within a column cannot be compared unless the sample numbers of the various types of terminals are taken into account. Terminals that formed multiple synapses with both GABAergic and non-GABAergic postsynaptic structures and terminals that formed synapses with an axon hillock are not included in this analysis. 
smaller (average diameter $\pm \mathrm{SD}, 11 \pm 2.6 \mu \mathrm{m}$ ) than the non-labelled neurons $(22.3 \pm 4.8 \mu \mathrm{m})$. These diameters agree with those of the small GABAergic neurons that project to the olive and with those of the large non-GABAergic neurons that have been shown both anatomically and physiologically to be excitatory and to project to the mesodiencephalic junction (Ruigrok and De Zeeuw, 1993; De

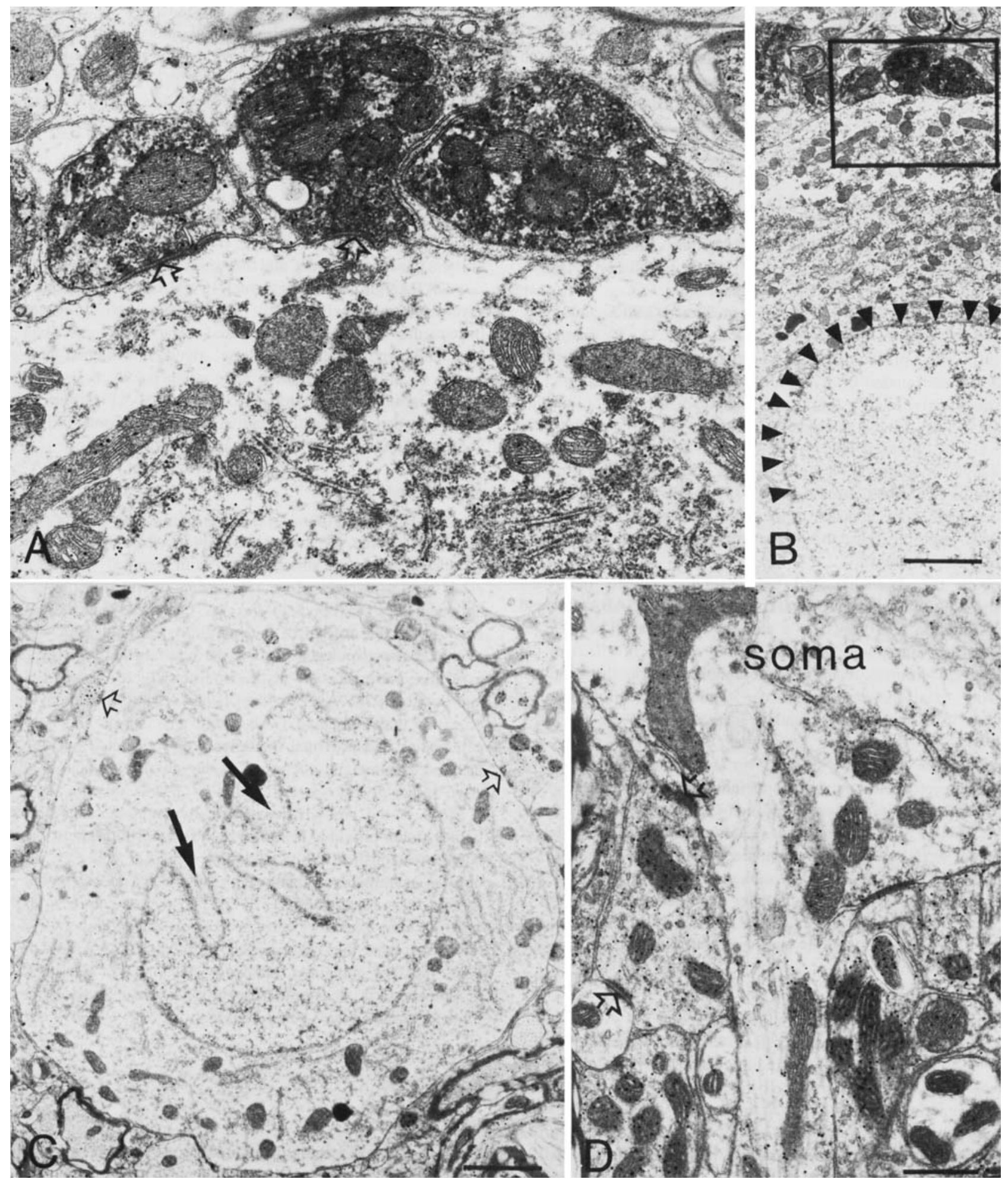

Fig. 3. Electron micrographs of GABAergic cell bodies contacted by GABAergic terminals. The medium-sized GABAergic neurons with smooth nuclei (filled triangles) were often contacted by L7-labelled terminals (A, B), while the somewhat smaller GABAergic neurons with indented nuclei (filled arrows) were only infrequently contacted by GABAergic terminals; relatively few of these GABAergic terminals were L7-positive (C, D). Open arrows indicate symmetrical synapses. Scale bars: B, $8 \mu \mathrm{m}$; C, $1.2 \mu \mathrm{m} ; \mathrm{D}, 0.42 \mu \mathrm{m}$. 
Zeeuw and Ruigrok, 1994; Teune et al., 1995). Since only a few of the small neurons were GABA-negative and glycine-negative, the number of false-negative cell bodies was probably limited. In contrast to the large neurons, which displayed numerous clumps of Nissl bodies and smooth nuclei, the smaller GABA-containing neurons often contained single cisterns of endoplasmic reticulum in their cytoplasm (see also Sotelo and Angaut, 1973). In general, both the inhibitory and excitatory neurons were rather diffusely distributed in all parts of the cerebellar and vestibular nuclei (for monkey see also Chan-Palay, 1977; cat, Walberg et al., 1990; rat, Batini et al., 1992; Chen and Hillman, 1993; Teune et al., 1995).

\section{Purkinje cell terminals}

The L7-labelled terminals ranged in diameter from 0.8 to $3.7 \mu \mathrm{m}$, as determined by serial section analysis, and usually included several mitochondria, many pleiomorphic vesicles, some coated vesicles and one or two dense core vesicles (Fig. 2). They established primarily symmetrical synapses; the larger terminals often formed more than one synapse. The Purkinje cell terminals contacted GABA-containing, glycine-containing or non-labelled neurons (Fig. 3A, B); frequently the cell bodies of the large non-labelled neurons were virtually completely surrounded by L7-positive terminals (see also Fig. 1B). In the quantitative analysis of the postsynaptic distribution of Purkinje cells in the lateral and interposed cerebellar nuclei $(n=986$; see Table 1), we observed that (i) the majority were apposed to nonGABAergic structures (79\%), and (ii) that about half of all Purkinje cell terminals contacted distal dendrites $(51 \%)$. In some cases GABAergic terminals were apposed to the axon hillock of GABAergic or non-GABAergic neurons; these few terminals were not included in the quantitative analysis.

In both the cerebellar and the vestibular nuclei, individual Purkinje cell terminals were found to innervate both GABAergic and non-GABAergic neurons (Figs 4 and 6). In non-serial sections of the lateral and interposed cerebellar nuclei 3\% (26 of 1012) of the Purkinje cell terminals made synaptic contacts with both structures. Following serial analysis of sets of $\sim 50$ ultrathin sections (with a thickness of $50 \mathrm{~nm}$ ), this percentage increased to $9 \%$ (34 of 378). Frequently, we encountered individual Purkinje cell axons that contained more than one terminal in a particular set of serial sections. Therefore, the percentage of individual Purkinje cell axons that contacted both GABAergic and non-GABAergic structures was higher (13\%; 49 of 378) than that of individual terminals. Considering that we did serial section analysis on pieces of tissue not thicker than $3 \mu \mathrm{m}$ and that individual Purkinje cell axons give off varicosities in the cerebellar and vestibular nuclei over hundreds of micrometres in the rostrocaudal plane (De Zeeuw et al., 1994b), it seems likely that most of the Purkinje cell axons innervate both GABAergic and nonGABAergic neurons.

\section{GABAergic terminals not derived from Purkinje cells}

The GABA-containing terminals that were not labelled for L7 resembled, apart from the lack of a diaminobenzidine reaction product, morphologically the Purkinje cell terminals. These L7-negative GABA-containing terminals, which must have been derived from interneurons and/or recurrent collaterals of GABAergic neurons projecting to the olive, contacted both GABAergic and non-GABAergic cell bodies and dendrites. Frequently, the L7-negative GABAcontaining terminals contacted the same GABAergic, glycinergic or non-labelled cell bodies or proximal dendrites as the Purkinje cell terminals and non-labelled terminals did (Figs 2A, B and 5C). In general, we did not find significant differences between the postsyn- aptic distribution of the L7-negative terminals and the Purkinje cell terminals (Table 1). In some instances, however, small GABAcontaining neurons were contacted by relatively few terminals; most of these terminals were immunonegative for L7 despite adequate penetration of the $\mathrm{L} 7$ antibody in the uppermost $3 \mu \mathrm{m}$ of the tissue (Fig. 3C, D). These small GABA-containing neurons frequently contained nuclei that were invaginated, and they may correspond to the small neurons observed by Matsushita and Iwahori (1971) and Van Der Want (1986) and Van Der Want and Voogd (1987) in the cerebellar nuclei of the cat; these neurons receive few axosomatic terminals, some of which are derived from recurrent collaterals from cerebellar projection neurons.

\section{Glycinergic terminals}

The glycine-containing terminals were generally of the same size as the Purkinje cell terminals (Fig. 5), but more often they contained clusters of neurofilaments, and they showed a preference for contacting non-GABAergic and non-glycinergic cell bodies and dendrites. The glycinergic terminals usually formed symmetrical synapses, but infrequently they established mixed synapses, as described below. Frequently, individual GABAergic, glycinergic or non-labelled cell bodies or proximal dendrites were contacted by both glycine-containing terminals and Purkinje cell terminals (Fig. 5D). We were unable to demonstrate that individual glycine-containing terminals contacted both an inhibitory and an excitatory cell.

\section{Non-labelled terminals}

The non-labelled terminals, i.e. the terminals that did not contain L7, GABA or glycine, showed the morphology characteristic of excitatory terminals: they were usually filled with rounded vesicles and they established asymmetrical synapses, some of which displayed subsynaptic densities (Taxi bodies) (Fig. 2A, C). The size of these terminals was comparable to that of the inhibitory terminals, but with regard to their postsynaptic distribution the non-labelled terminals showed a relative preference for distal dendrites (Table 1; see also Angaut and Sotelo, 1973; Van Der Want, 1986). Even so, in many cases we observed that individual GABAergic, glycinergic and non-labelled cell bodies and proximal dendrites were contacted by both a nonlabelled terminal and a terminal of the inhibitory type, i.e. a GABAergic and/or glycinergic terminal (Figs 2A, C and 4B).

Some of the non-labelled terminals contacting non-labelled cell bodies and/or proximal dendrites in the medial and superior vestibular nucleus established mixed excitatory synapses, i.e. they established both an asymmetrical chemical synapse and an electrotonic contact formed by a gap junction (Fig. 5A, B) (see also Sotelo and Palay, 1970). These excitatory terminals were usually larger (3-5 $\mu \mathrm{m}$ in diameter) than the non-labelled terminals apposed to the distal dendrites, they contained rounded vesicles and an abundant number of mitochondria, and they frequently showed a web of fine neurofilaments in the centre of the terminal.

\section{Discussion}

\section{Light microscopy}

The L7 immunoreactivity of terminal profiles was present in all parts of the cerebellar and vestibular nuclei, which agrees with the well known ubiquitous innervation of these nuclei by the Purkinje cells (for reviews see Chan-Palay, 1977; Voogd and Bigaré, 1980). In addition, we observed that L7 was present in the nucleus prepositus hypoglossi. The projection from the flocculus to this nucleus is 

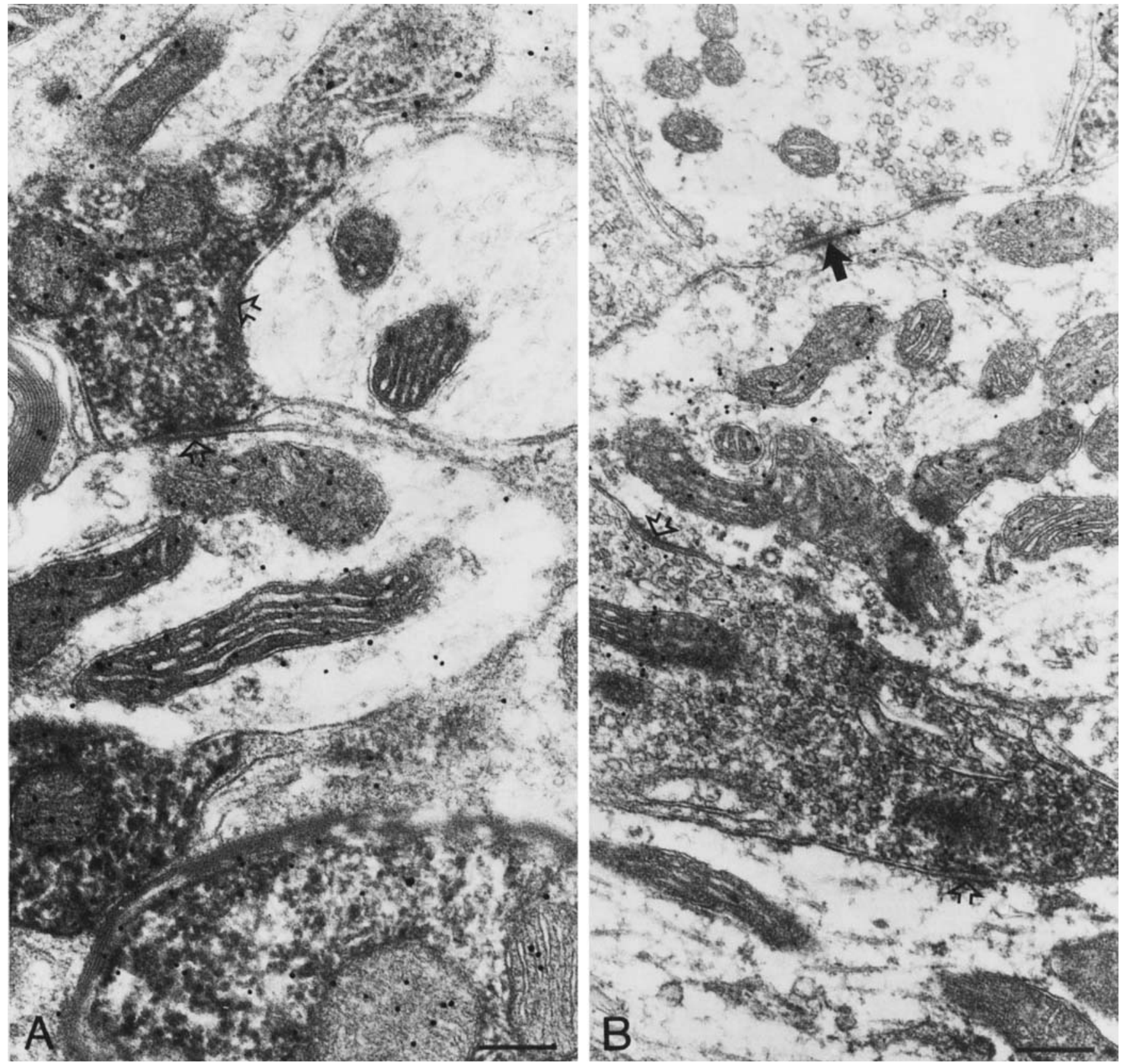

FIG. 4. Both in the vestibular (A) and cerebellar (B) nuclei individual Purkinje cell terminals were found to innervate both GABAergic and non-GABAergic dendrites. Note that the GABAergic dendrite in B is contacted by both an L7-labelled GABAergic dendrite and an excitatory, non-GABAergic terminal with round vesicles and an asymmetrical synapse. Open arrows indicate symmetrical synapses and the filled arrow indicates an asymmetrical synapse. Scale bars: A, $0.19 \mu \mathrm{m} ; \mathrm{B}, 0.25 \mu \mathrm{m}$.

debated (rabbit, Tan et al., 1995; De Zeeuw et al., 1994b; rat, Umetani, 1992; cat, Angaut and Brodal, 1967; Baker and Berthoz, 1975; Yingcharoen and Rinvik, 1983; primates, Haines, 1977) and the projection from folium $p$ of the ventral paraflocculus to the nucleus prepositus hypoglossi has only been demonstrated with the use of retrograde tracing (rabbit, Yamamoto, 1978). Because the medial vestibular nucleus adjacent to the nucleus prepositus hypoglossi also receives a projection from folium p (rabbit, Tan et al., 1995), this latter finding could have been biased. The present study demonstrates that the Purkinje cell input to the nucleus prepositus hypoglossi is sparse but present, and that the Purkinje cell terminals make synaptic contacts with both the inhibitory and the presumptive excitatory neurons in this nucleus.

\section{Electron microscopy}

GABAergic and non-GABAergic targets of Purkinje cells

The major findings of this study are that individual Purkinje cells contact neurons with a wide range of sizes and shapes and that these neurons can be GABAergic and non-GABAergic (Fig. 6). The vast majority of the GABAergic neurons contacted by the Purkinje cells in the cerebellar nuclei are probably neurons projecting to the inferior 

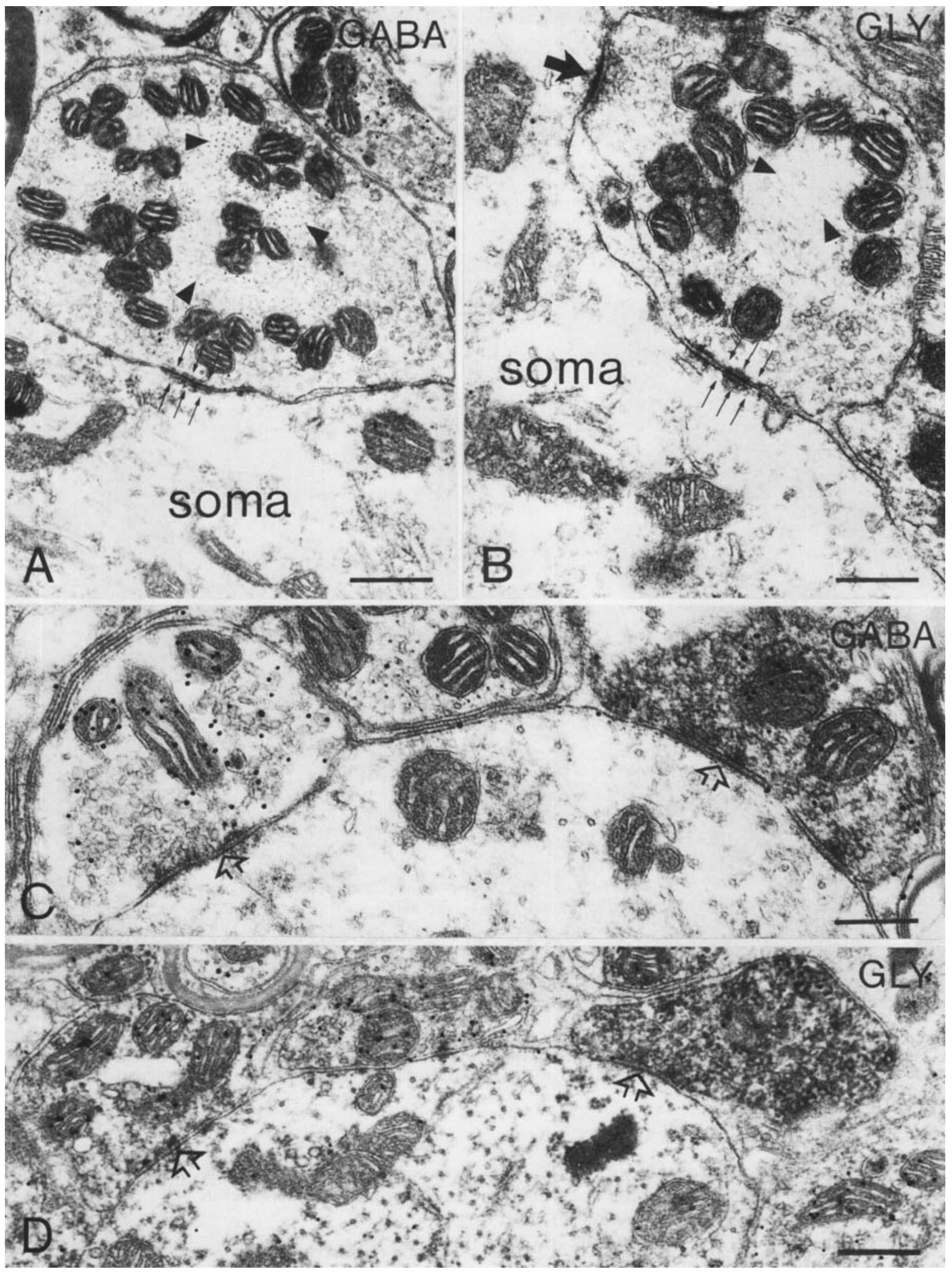

Fig. 5. Electron micrographs of terminals in the medial (A, D) and superior (B, C) vestibular nuclei. Some of the excitatory terminals in these vestibular nuclei had so-called mixed synapses (Sotelo and Palay, 1970), i.e. these terminals contained both asymmetrical synapses (filled arrow) and gap junctions (small arrows). Frequently the terminals with mixed synapses contained a cluster of neurofilaments in the centre of the terminal (triangles). The dendrites in the vestibular nuclei were often contacted by L7-labelled terminals, L7-negative GABAergic terminals (C) and glycinergic (GLY) terminals (D). Following serial section analysis the glycinergic 'terminals' in D were found to be part of the same glycinergic fibre (data not shown). Scale bars: A, $0.42 \mu \mathrm{m}$; B, $0.39 \mu \mathrm{m}$; $\mathrm{C}, 0.24 \mu \mathrm{m} ; \mathrm{D}, 0.35 \mu \mathrm{m}$. 


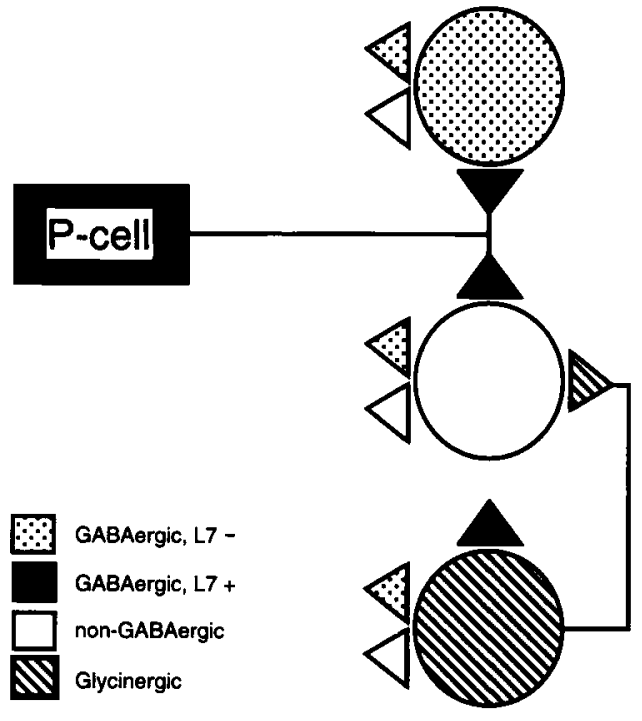

FIG. 6. Diagram of major connections in the cerebellar and vestibular nuclei. Individual Purkinje cell terminals innervated both GABAergic and nonGABAergic neurons. Triangles indicate terminals, circles indicate postsynaptic structures.

olive (De Zeeuw et al., 1989; Nelson and Mugnaini, 1989), since Fredette and Mugnaini (1991) observed that at least $93 \%$ of glutamic acid decarboxylase-positive neurons in the cerebellar nuclei are retrogradely labelled following injection of horseradish peroxidase in the inferior olive, and that these neurons are innervated by GABAergic terminals in the same fashion as they are innervated by the L7 terminals. This presumably monosynaptic input from the Purkinje cells onto the olivary projection neurons in the cerebellar nuclei indicates that the olivocerebellar modules described by Voogd and Bigare (1980) are indeed formed by a three-element loop consisting of a specific olivary subnucleus, a specific cerebellar sagittal zone of Purkinje cells, and a specific cerebellar nucleus. The finding that the Purkinje cell terminals also synapsed onto the GABAergic neurons in group $y$, the nucleus prepositus hypoglossi, and the descending and medial vestibular nucleus, which are known to project to the ventrolateral outgrowth, dorsal cap, and $\beta$-nucleus of the inferior olive respectively (De Zeeuw et al., 1993 and 1994a), suggests that the arrangement of the three-element modules also occurs in the vestibulocerebellum (De Zeeuw et al., 1994b; Tan et al., 1995). The observation that individual Purkinje cells innervate both GABAergic and non-GABAergic neurons in the cerebellar and vestibular nuclei suggests that the Purkinje cells may control simultaneously the inhibitory feedback to the inferior olive, which is part of the closed, three-element, anatomical pathway, and the excitatory cerebellar output system, which is part of an open anatomical pathway through which the motor commands are finally influenced (De Zeeuw et al., 1994b). These combined inhibitory and excitatory effects of individual Purkinje cells may, therefore, be of crucial importance for the role of the cerebellum in controlling the timing of movements (Welsh et al., 1995; De Zeeuw et al., 1995).

\section{Purkinje cell input to glycinergic neurons}

Apart from the large glycinergic neurons that occur in the fastigial nucleus and may contain aspartate, most of the glycinergic neurons in the cerebellar nuclei contain GABA as a co-neurotransmitter (Chen and Hillman, 1993). These glycine-containing neurons are probably not involved in the olivocerebellar modules since glycinergic boutons have not been found in the inferior olive (De Zeeuw et al., 1994a). Because no glycinergic projections from or to the cerebellar nuclei have been described and glycinergic terminals and receptors do occur in the nuclei (Chen and Hillman, 1993; present study), most of the glycine-containing neurons in the cerebellar nuclei may in fact be interneurons. Glycine and GABA could cooperate as inhibitory neurotransmitters that hyperpolarize target neurons by opening chloride channels (Curtis et al., 1968; Ottersen and Storm-Mathisen, 1990). The combination of the rapid inhibitory onset of glycine (Curtis et al., 1968) and the slower onset and longer duration by GABA (Faber and Korn, 1991) probably results in a rapidly evoked but sustained inhibition of postsynaptic targets. As such, activation of the glycinergic, GABAergic interneurons may, even though these neurons only provide a small proportion of the total of inhibitory terminals, result in temporal modulation of inhibitory patterns in the central nuclei neurons. The observations that Purkinje cells project not only to the GABAergic and non-GABAergic neurons but also to the glycinergic neurons, and that the glycinergic neurons were found to project mainly to the larger, presumptive excitatory neurons, may explain why the time course of the inhibition in the excitatory central nuclei neurons after climbing fibre activation does not correspond to the time course of the facilitation of the simple spikes after the climbing fibre pause (Ruigrok et al., 1994).

In the vestibular nuclei, glycine is also colocalized with other neurotransmitters like GABA and aspartate in the same neurons (Walberg et al., 1990). However, in these nuclei glycine is not restricted to interneurons since glycinergic neurons in the medial vestibular nucleus and descending vestibular nucleus have been shown to project to the abducens nucleus and spinal cord respectively (Spencer et al., 1989, 1992; Shinoda et al., 1992).

\section{Non-Purkinje cell terminals with mixed synapses}

Some of the non-labelled terminals and some of the glycinergic terminals in the medial and superior vestibular nucleus formed mixed excitatory synapses establishing both an asymmetrical chemical synapse and an electrotonic contact formed by a gap junction with a non-labelled cell body. Similar terminals have been described by Sotelo and Palay (1970) for the ventral part of the lateral vestibular nucleus [currently named the magnocellular medial vestibular nucleus (De Zeeuw et al., 1994b)]. Since we were unable to find terminals with these mixed synapses in the cerebellar nuclei or nucleus prepositus hypoglossi, it is possible that they are in fact terminals of primary vestibular afferents. Morphologically, these terminals would correspond best to those of the irregular primary afferents (Sato and Sasaki, 1993; see also Korte and Friedrich, 1979). If the terminals of the primary afferents indeed make electrotonic contacts with the cell bodies of second-order vestibular neurons, then these axosomatic gap junctions may explain why floccular activity can be evoked by stimulation of the vestibular labyrinths at latencies suggestive of a monosynaptic connection (Shinoda and Yoshida, 1975; Ito, 1984).

\section{Acknowledgements}

The authors would like to thank Mr E. Dalm, Mr H. van der Burg, Mr R. Hawkins, and Mrs E. Goedknegt for their excellent technical assistance. This research was supported in part by grants from NWO ( $R$ 95-260; Dutch Organization for Fundamental Research) and KNAW (Royal Dutch Academy of Sciences). 


\section{Abbreviations}

GABA

$\gamma$-amino butyric acid

\section{References}

Aas, J. E., and Brodal, P. (1990) GABA and glycine as putative transmitters in subcortical pathways to the pontine nuclei. A combined immunocytochemical and retrograde tracing study in the cat with some observations in the rat. Neuroscience, 34, 149-162.

Angaut, P. and Brodal, A. (1967) The projection of the 'vestibulocerebellum' onto the vestibular nuclei in the cat. Arch. Ital. Biol., 105, 441-479.

Angaut, P. and Sotelo, C. (1973) The fine structure of the cerebellar central nuclei in the cat. II. Synaptic organization. Exp. Brain Res., 16, 431-454.

Baker, R. and Berthoz, A. (1975) Is the prepositus hypoglossi nucleus the source of another vestibulo-ocular pathway? Brain Res., 86, 121-127.

Barmack, N. H., Mugnaini, E. and Nelson, B. J. (1989) Vestibularly-evoked activity of single neurons in the $\beta$-nucleus of the inferior olive. In Strata, P. (ed.), The Olivocerebellar System in Motor Control. Springer-Verlag, Berlin. Exp. Brain Res., Suppl. 17, 313-323.

Batini, C., Compoint, C., Buisseret-Delmas, Daniel, C. H. and Guegan, M. (1992) Cerebellar nuclei and the nucleocortical projections in the rat: retrograde tracing coupled to GABA and glutamate immunohistochemistry. J. Comp. Neurol., 315, 74-84.

Berrebi, A. S. and Mugnaini, E. (1992) Characteristics of labeling of the cerebellar Purkinje neuron by $\mathrm{L} 7$ antiserum. J. Chem. Neuroanat., 5, 235-243.

Berrebi, A. S., Oberdick, J., Sangameswaran, L., Christakos, S., Morgan, J. I. and Mugnaini, E. (1991) Cerebellar Purkinje cell markers are expressed in retinal bipolar neurons. J. Comp. Neurol., 308, 630-649.

Buijs, R. M., van Vulpen, E. H. S. and Geffard, M. (1987) Ultrastructural localization of GABA in the supraoptic nucleus and the neural lobe. Neuroscience, 20, 347-355.

Chan-Palay, V. (1977) Cerebellar Dentate Nucleus. Organization. Cytology and Transmitters. Springer-Verlag, Berlin.

Chen, S. and Hillman, D. E. (1993) Colocalization of neurotransmitters in the deep cerebellar nuclei. J. Neurocytol., 22, 81-91.

Curtis, D. R., Hosli, L., Johnston, G. A. R. and Johnston, I. H. (1968) The hyperpolarization of spinal motorneurons by glycine and related amino acids. Exp. Brain Res., 5, 235-258.

De Zeeuw, C. I. and Ruigrok, T. J. H. (1994) Olivary projecting neurons in the nucleus of Darkschewitsch in the cat receive excitatory monosynaptic input from the cerebellar nuclei. Brain Res., 653, 345-350.

De Zeeuw, C. I., Holstege, J. C. Ruigrok, T. J. H. and Voogd, J. (1989) An ultrastructural study of the GABAergic, the cerebellar and the mesodiencephalic innervation of the cat medial accessory olive: anterograde tracing combined with immunocytochemistry. J. Comp. Neurol., 284, 12-35.

De Zeeuw, C. I., Wentzel, P. and Mugnaini, E. (1993) Fine structure of the dorsal cap of the inferior olive and its GABAergic and non-GABAergic input from the nucleus prepositus hypoglossi in rat and rabbit. J. Comp. Neurol., 327, 63-82.

De Zeeuw, C. I., Gerrits, N. M., Voogd, J., Leonard, C. and Simpson, J. L.(1994a) The rostral dorsal cap and ventrolateral outgrowth of the rabbit inferior olive receive a GABAergic input from dorsal group $y$ and the ventral dentate nucleus. J. Comp. Neurol., 341, 420-432.

De Zeeuw, C. I., Wylie, D. R., DiGiorgi, P. L. and Simpson, J. I. (1994b) Projections of individual Purkinje cells of identified zones in the flocculus to the vestibular and cerebellar nuclei in the rabbit. J. Comp. Neurol., 349, 428-448.

De Zeeuw, C. I., Wylie, D. R., Stahl, J. S. and Simpson, J. I. (1995) Phase relations of Purkinje cells in the rabbit flocculus during compensatory eye movements. J. Neurophysiol., in press.

Faber, D. S. and Korn, H. (1991) Do some M-cell glycine receptors interact with GABA? Soc. Neurosci. Abstr, 17, 246.

Fredette, B. J. and Mugnaini, E. (1991) The GABAergic cerebello-olivary projection in the rat. Anat. Embryol., 184, 225-243.

Fukushima, K., Peterson, B. W., Uchino, Y., Coulter, J. D. and Wilson, V. J. (1977) Direct fastigiospinal fibers in the cat. Brain Res., 126, 538-542.

Haines, D. E. (1977) Cerebellar corticonuclear and corticovestibular fibers of the flocculonodular lobe in a prosimian primate (Galago senegalensis). J. Comp. Neurol., 174, 607-630.
Highstein, S. M. (1973) The organization of the vestibulo-ocular and trochlear reflex pathways in the rabbit. Exp. Brain Res., 17, 285-300.

Highstein, S. M. and Reisine, H. (1979) Synaptic and functional organization of vestibulo-ocular reflex pathways. In Granit, $\mathbf{R}$. and Pompeiano, $\mathbf{O}$. (eds), Reflex Control of Posture and Movement. Progress in Brain Research, Vol. 50. Elsevier, Amsterdam, pp. 431-442.

Ito, M. (1984) The Cerebellum and Neural Control. Raven Press, New York. Ito, M., Nisimaru, N. and Yamamoto, M. (1976) Pathways for the vestibuloocular reflex excitation arising from semicircular canals of rabbits. Exp. Brain Res., 24, 257-271.

Kevetter, G. A. and Hoffman, R. D. (1991) Excitatory amino acid immunoreactivity in vestibulo-ocular neurons in gerbils. Brain Res., 554, 348-351.

Korte, G. E. and Friedrich, V. L. (1979) The fine structure of the feline superior vestibular nucleus: identification of the primary vestibular afferents. Brain Res., 176, 3-32.

Matsushita, M, and Iwahori, N. (1971) Structural organization of the interpositus and the dentate nuclei. Brain Res., 35, 17-36.

Nelson, B. J. and Mugnaini, E. (1989). Origins of GABAergic inputs to the inferior olive. In Strata, P. (ed.), The Olivocerebellar System in Motor Control. Springer-Verlag, Berlin. Exp. Brain Res., Suppl. 17, 86-107.

Oberdick, J., Levinthal, F. and Levinthal, C. (1988) A Purkinje cell differentiation marker shows a partial DNA sequence homology to the cellular sis/PDGF2 gene. Neuron, 1, 367-376 [erratum 3, 385].

Ottersen, O. P. and Storm-Mathisen, J. (eds) (1990) Glycine Neurotransmission. John Wiley.

Palkovits, M., Mezey, E., Hámori, J. and Szentágothai, J. (1977) Quantitative histological analysis of the cerebellar nuclei in the cat. I. Numerical data on cells and on synapses. Exp. Brain Res., 28, 189-209.

Ruigrok, T. J. H. and De Zeeuw, C. I. (1993) Electron microscopy of in vivo recorded and intracellularly injected inferior olivary neurons and their GABAergic innervation in the cat. J. Microsc. Res. Technol., 24, 85-102.

Ruigrok, T. J. H., Teune, T. M., Van Der Burg, J. and Voogd, J. (1994) Electrical stimulation of the inferior olive results in enhanced activity of projection neurons in the cerebellar nuclei of the ketamine anesthetized rat. Soc. Neurosci. Abstr., 71, 1.9, p. 1744

Sato, F., and Sasaki, H.(1993) Morphological correlations between spontaneously discharging primary vestibular afferents and vestibular nucleus neurons in the cat. J. Comp. Neurol., 333, 554-566.

Seguela, P., Geffard, M., Buijs, R. and Moal, M. (1984) Antibodies against gamma-aminobutyric acid: specificity studies and immunocytochemical results. Proc. Natl Acad. Sci. USA, 81, 3888-3892.

Shinoda, Y. and Yoshida, K. (1975) Neural pathways from the vestibular labyrinths to the flocculus in the cat. Exp. Brain Res., 22, 97-111.

Shinoda, Y., Kakei, S., Sugiuchi, Y. and Kawasaki, T. (1992) Morphological features of single inhibitory medial vestibulospinal axons receiving horizontal semicircular canal input. Soc. Neurosci. Abstr., 18, 216.2.

Sotelo, C. and Angaut, P. (1973) The fine structure of the cerebellar central nuclei in the cat I. Neurons and neuroglial cells. Exp. Brain Res., 16, $410-430$.

Sotelo, C. and Palay, S. L. (1970) The fine structure of the lateral vestibular nucleus in the rat. II. Synaptic organization. Brain Res., 18, 93-115.

Spencer, R. F., Wenthold, R. J. and Baker, R. (1989) Evidence for glycine as an inhibitory neurotransmitter of vestibular, reticular, and prepositus hypoglossi neurons that project to the cat abducens nucleus. J. Neurosci., 9. 2718-2736

Spencer, R. F., Wang, S. and Baker, R. (1992) The pathways and functions of GABA in the oculomotor system. Progr. Brain Res., 90, 307-331.

Tan, J., Epema, A. H. and Voogd, J. (1995) Zonal organization of the flocculovestibular nucleus projection in the rabbit: a combined axonal tracing and acetylcholinesterase histochemical study. J. Comp. Neurol., 356, $51-71$

Teune, T. M., Van Der Burg, J. and Ruigrok, T. J. H. (1995) Cerebellar projections to the red nucleus and inferior olive originate from separate populations of neurons in the rat: a non- fluorescent double labeling study. Brain Res., in press.

Umetani, T. (1992) Efferent projections from the flocculus in the albino rat as revealed by an autoradiographic orthograde tracing method. Exp. Brain Res., 586, 91-103.

Van der Want, J. J. L. (1986) Cerebellar circuitry, identification of mossy and climbing fiber terminals and their synapses in the cerebellum of the cat. Thesis, Rodopi, Amsterdam.

Van Der Want, J. J. L. and Voogd, J. (1987) Ultrastructural identification and 
localization of climbing fiber terminals in the fastigial nucleus of the cat. J. Comp. Neurol., 258, 281-290.

Voogd, J., and Bigaré, F. (1980) Topographical distribution of olivary and corticonuclear fibers in the cerebellum. A review. In Courville, J., de Montigny, C. and Lamarre, Y. (eds), The Inferior Olivary Nucleus. Raven Press, New York, pp. 207-235.

Walberg, F., Ottersen, O. P. and Rinvik, E. (1990) GABA, glycine. aspartate, glutamate, and taurine in the vestibular nuclei: an immunocytochemical investigation in the cat. Exp. Brain Res., 79, 547-563.

Welsh, J. P., Lang, E., Sugihara, J. I. and Llinás, R. R. (1995) Dynamic organization of motor control within the olivocerebellar system. Nature, 374, 453-457.

Wenthold, R. J., Huie, D., Altschuler, R. A. and Reeks, K. A. (1987) Glycine immunoreactivity localized in the cochlear nucleus and superior olivary complex. Neuroscience, 22, 897-912.
Wentzel, P. R., De Zeeuw, C. I., Holstege, J. C. and Gerrits, N. M. (1995) Inhibitory and excitatory synaptic inputs to the oculomotor inputs from VOR related nuclei in the rabbit. Neuroscience, 65, 161-174.

Wilson, V. J., Uchino, Y., Susswein, A. and Fukushima, K. (1977) Properties of direct fastigiospinal fibers in the cat. Brain Res., 126, 543-546.

Yamamoto, M. (1978) Localization of rabbit's flocculus Purkinje cells projecting to the cerebellar lateral nucleus and the nucleus prepositus hypoglossi investigated by means of the horseradish peroxidase retrograde axonal transport. Neurosci. Lett., 7, 197-202.

Yamamoto, M., Shimoyama, I. and Highstein, S. M. (1978) Vestibular nucleus neurons relaying excitation from the anterior canal to the oculomotor nucleus. Brain Res., 148, 31-42.

Yingcharoen, K. and Rinvik, E. (1983) Ultrastructural demonstration of a projection from the flocculus to the nucleus prepositus hypoglossi in the cat. Exp. Brain Res., 51, 192-198. 